\title{
TEMPERATURA DEL AIRE EN LA REGIÓN METROPOLITANA DE BARCELONA A PARTIR DE LA TEMPERATURA EN SUPERFÍCIE MODIS Y DE DATOS TOPOGEOGRÁFICOS
}

\section{AIR TEMPERATURE IN THE METROPOLITAN REGION OF BARCELONA FROM THE SURFACE TEMPERATURE MODIS AND TOPOGEOGRAPHIC DATA}

\author{
SERRA DE LAROCHA, Carina \\ Universidad Politécnica de Catalunya (UPC) \\ Departamento de Física (FIS) \\ Profesora Titular de Universidad \\ Av. Diagonal 647, 08028. Barcelona, España \\ Correo electrónico: carina.serra@upc.edu \\ Teléfono: +34 934015934
}

\author{
LANA PONS, Xavier \\ Universidad Politécnica de Catalunya (UPC) \\ Departamento de Física (FIS) \\ Profesor Titular de Universidad \\ Av. Diagonal 647, 08028. Barcelona, España \\ Correo electrónico: francisco.javier.lana@upc.edu \\ Teléfono: +34 934010825
}

\section{MARTÍNEZ SANTAFÉ, Maria Dolors}

Universidad Politécnica de Catalunya (UPC)

Departamento de Física (FIS), Centro de Política de Suelo y Valoraciones (CPSV)

Profesora Titular de Universidad

Av. Diagonal 649, 08028. Barcelona, España

Correo electrónico: dolors.martinez@upc.edu

Teléfono: +34 934016378

\section{ROCA, Josep}

Universidad Politécnica de Catalunya (UPC)

Departamento de Tecnología de la Arquitectura (TA), Centro de Política de Suelo y Valoraciones (CPSV) Catedrático de Universidad

Av. Diagonal 649, 08028. Barcelona, España

Correo electrónico: josep.roca@upc.edu

Teléfono: +34 934016396

\section{ARELLANO, Blanca}

Universidad Politécnica de Catalunya (UPC)

Departamento de Tecnología de la Arquitectura (TA), Centro de Política de Suelo y Valoraciones (CPSV)

Profesora asociada y Personal de Investigación (PSR)

Av. Diagonal 649, 08028 Barcelona, España

Correo electrónico: blanca.arellano@upc.edu

Teléfono: +34 934054383

\section{BIERE, Rolando}

Universidad Politécnica de Catalunya (UPC)

Departamento de Tecnología de la Arquitectura (TA), Centro de Política de Suelo y Valoraciones (CPSV)

Profesor asociado y Personal de Investigación (PSR)

Av. Diagonal 649, 08028. Barcelona, España

Correo electrónico: rolando.biere@upc.edu

Teléfono: +34 934016396

Citación: SERRA DE LAROCHA, C. et al. Temperatura del aire en la Región Metropolitana de Barcelona a partir de la temperatura en superfície MODIS y de datos topogeográficos. En: Libro de proceedings, CTV 2018. XII Congreso Internacional Ciudad y Territorio Virtual. "Ciudades y Territorios Inteligentes". UNCuyo, Mendoza, 5-7 septiembre 2018. Barcelona: CPSV, 2018, p. 357-368. 


\section{MOIX BERGADÁ, Montserrat}

Universidad Politécnica de Catalunya (UPC)

UTG Ámbito Arquitectura Barcelona (UTGAB)

Personal de investigación (PAS)

Av. Diagonal 649, 08028 Barcelona, España

Correo electrónico: monserrat.moix@upc.edu

Teléfono: +34 934010760

Palabras Clave: MODIS; temperatura del aire; NDVI; NDBI; Regresión Múltiple; Región Metropolitana de Barcelona

Key words: MODIS; air temperature; NDVI; NDBI; Multiple Regression; Barcelona Metropolitan Region

\section{Resumen}

La Región Metropolitana de Barcelona (RMB), es una de las áreas del Mediterráneo más densamente pobladas. Por ello, tiene mucho interés conocer con detalle la temperatura del aire, ya que, en situaciones extremas, como olas de frío o de calor, puede afectar a la salud y calidad de vida de la población. Puesto que el número de estaciones termométricas que hay en la actualidad es insuficiente para realizar una interpolación óptima, se opta por estimar la temperatura del aire a partir de la temperatura en superficie, proporcionada por el satélite MODIS, y de otras variables como los índices de vegetación y de edificación y variables topogeográficas. Para ello se ha realizado la Regresión Múltiple en la que las variables dependientes son la temperatura mínima, media y máxima diaria del aire de 48 estaciones termométricas. Las variables independientes de la regresión son: las temperaturas en superficie de día y de noche, los índices de vegetación NDVI y de edificación NDBI, todas ellas correspondientes al año 2015, y variables topogeográficas como la latitud, longitud, distancia al mar, altitud, pendiente y orientación. Además, también se ha tenido en cuenta el día del calendario. De los resultados obtenidos en la regresión múltiple se deduce que las variables que más importancia tienen en la ecuación son: la temperatura en superficie nocturna, la latitud, la longitud y día del calendario, para el caso de la temperatura mínima; las temperaturas nocturna y diurna en superficie para la temperatura media, y las temperaturas nocturna y diurna en superficie, la latitud, la longitud y la altitud, para la temperatura máxima. Los coeficientes de regresión al cuadrado obtenidos son de 0.92 para la temperatura mínima y la máxima y de 0.96 para la temperatura media. Los errores cuadráticos medios obtenidos son de $1.93{ }^{\circ} \mathrm{C}$ para la temperatura mínima, de $1.96{ }^{\circ} \mathrm{C}$ para la temperatura media y de $2.04{ }^{\circ} \mathrm{C}$ para la máxima. A partir de la regresión múltiple se puede representar la distribución espacial de temperatura diaria de la RMB con una resolución de $1 \mathrm{~km}^{2}$, que es la que proporciona el satélite MODIS.

\section{Abstract}

The Metropolitan Region of Barcelona (RMB), is one of the most densely populated areas of the Mediterranean. Therefore, it is very interesting to know in detail the air temperature, since in extreme situations, such as cold or heat waves, it can affect the health and life quality of the population. Since the number of thermometric stations currently available is insufficient for optimal spatial interpolation, we choose to estimate the air temperature based on the surface temperature provided by the MODIS satellite, and other variables such as the indices of

Citación: SERRA DE LAROCHA, C. et al. Temperatura del aire en la Región Metropolitana de Barcelona a partir de la temperatura en superfície MODIS y de datos topogeográficos. En: Libro de proceedings, CTV 2018. XII Congreso Internacional Ciudad y Territorio Virtual. "Ciudades y Territorios Inteligentes". UNCuyo, Mendoza, 5-7 septiembre 2018. Barcelona: CPSV, 2018, p. 357-368. 
vegetation and building and topo geographic variables. For this purpose, Multiple Regression has been performed, taking as dependent variables the minimum, mean and maximum daily air temperatures of 48 thermometric stations. The independent variables of the regression are: the day and night surface temperatures, the NDVI and NDBI indices, all of them corresponding to the year 2015, and topo geographic variables such as latitude, longitude, distance to the sea, altitude, slope and orientation. In addition, the calendar day has also been taken into account. From the results obtained in the multiple regression, it can be deduced that the most important variables in the equation are: the temperature at night, latitude, longitude and calendar day, for the case of the minimum temperature; the night and day temperatures on the surface for the average temperature, and the night and day temperatures on the surface, latitude, longitude and altitude, for the maximum temperature. The squared regression coefficients obtained are 0.92 for the minimum and maximum temperature and 0.96 for the average temperature. The average square errors obtained are $1.93 \stackrel{\circ}{\circ} \mathrm{C}$ for the minimum temperature, $1.96{ }^{\circ} \mathrm{C}$ for the average temperature and $2.04 \stackrel{\circ}{\circ} \mathrm{C}$ for the maximum. After applying the multiple linear regressions, the spatial distribution of the daily temperature of the RMB can be represented with a resolution of $1 \mathrm{~km}^{2}$, which is the provided by the MODIS satellite.

\section{Introducción}

La estimación de la temperatura del aire $\mathrm{T}_{\mathrm{a}}$ (aproximadamente a $2 \mathrm{~m}$ de altura) a partir de la temperatura en superficie LST proporcionada por el satélite MODIS $\left(1 \mathrm{~km}^{2}\right.$ de resolución) es una opción últimamente bastante utilizada (Zaksek et al. 2009) debido a la escasez de estaciones termométricas que hacen difícil las interpolaciones de los valores de temperatura del aire. El método más simple consiste en la relación lineal entre las temperaturas $T_{a}$ y LST (Fu et al., 2011; Sohrabinia et al., 2015). En otros trabajos se realiza el estudio para los los diferentes tipos de terreno (Shen and Leptoukh, 2011) o se incluye el índice de Vegetación NDVI (Vancutsem et al, 2010; Cristóbal, et al. 2008; Nieto et al., 2011; Wenbin et al. 2013; Bustos and Meza, 2015). Estudios más complejos que aplican la regresión múltiple, incluyen también la radiación solar o el albedo (Xu et al., 2014), o variables geográficas y topográficas como latitud, altitud, distancia al mar y la pendiente o el día del calendario (Benali et al., 2012; Recondo et al., 2013; Peón et al., 2014 Good, 2015; Yang et al., 2017, Janatian et al. 2017).

El objetivo de este trabajo es estimar la temperatura del aire de la Región Metropolitana de Barcelona mediante la regresión múltiple y la detección de las variables qué más intervienen en ella. Las aplicaciones que se pueden dar posteriormente son múltiples. Una de ellas podría ser la detección de las islas de calor urbano (UHI), su distribución espacial e intensidad.

\section{Base de datos y zona de estudio}

La Región Metropolitana de Barcelona (RMB), con una extensión de $3242.2 \mathrm{~km}^{2}$, es una de las áreas del Mediterráneo con mayor población. Concretamente, la ciudad de Barcelona, tiene una densidad aproximada de 16000 habitantes $/ \mathrm{km}^{2}$. La RMB se encuentra en el NE de la Península Ibérica, y está delimitada por el Mar Mediterráneo y la Cordillera Prelitoral catalana, con altitudes de hasta $1700 \mathrm{~m}$.

Para el estudio de la temperatura del aire se han considerado la temperatura diaria mínima, $\mathrm{T}_{\min }$, media, $\mathrm{T}_{\text {mean, y }}$ máxima $\mathrm{T}_{\max }$ de 48 estaciones termométricas (Figura 1) correspondientes

Citación: SERRA DE LAROCHA, C. et al. Temperatura del aire en la Región Metropolitana de Barcelona a partir de la temperatura en superfície MODIS y de datos topogeográficos. En: Libro de proceedings, CTV 2018. XII Congreso Internacional Ciudad y Territorio Virtual. "Ciudades y Territorios Inteligentes". UNCuyo, Mendoza, 5-7 septiembre 2018. Barcelona: CPSV, 2018, p. 357-368. 
al año 2015, proporcionadas por el Servei Meteorològic de Catalunya (SMC) y la Agencia Estatal de Meteorología (AEMET). En la figura se pueden ver cinco estaciones fuera de la región pero que se han considerado dada su proximidad. También se representa la altitud con una resolución de $1 \mathrm{~km}^{2}$.

Figura 1. Mapa de las estaciones y altitud de la RMB

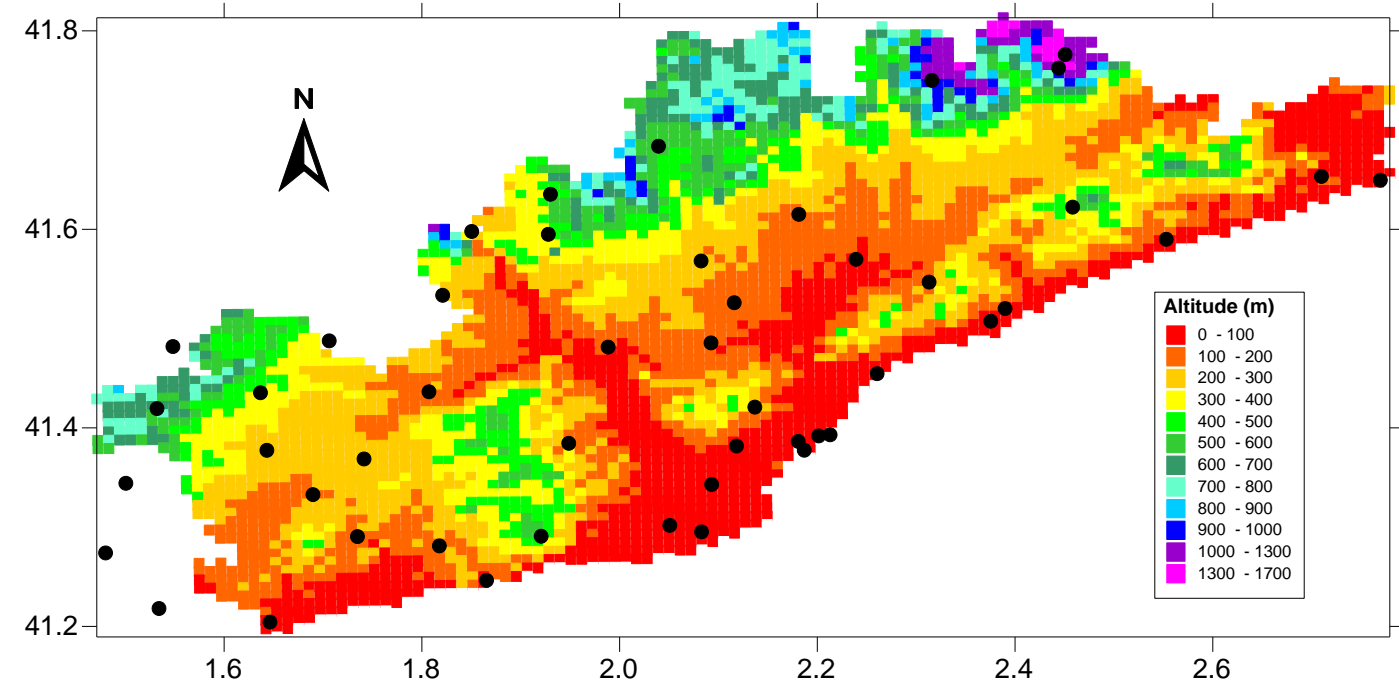

Fuente: Elaboración propia

Las temperaturas diarias LST de MODIS se han obtenido a partir del producto MOD11A1 que incluye la temperatura de dia LSTd (10:30 UTC) y la de noche LSTn (22:30 UTC), con una resolución de $1 \mathrm{~km}^{2}$. El índice de vegetación NDVI se ha extraído del producto MOD13Q1, con un promedio de 16 días y una resolución de $250 \mathrm{~m}$. El índice de edificación NDBI se ha calculado a partir de las bandas 6 (MIR) y 2 (NIR) del producto MOD09A1 con un promedio de 8 días y una resolución de $500 \mathrm{~m}$. El 50\% de las estaciones utilizadas presentan valores de $\mathrm{NDVI}$ entre 0.3 y 0.5 , correspondientes a emplazamientos con poca vegetación. Con respecto a los valores de NDBI la mayoría de estaciones tiene valores entre -0.2 y 0.1 . En la Figura 2 se muestra el histograma de valores de NDVI y de NDBI.

Figura 2: Histograma de valores de NDVI y de NDBI de las estaciones utilizadas
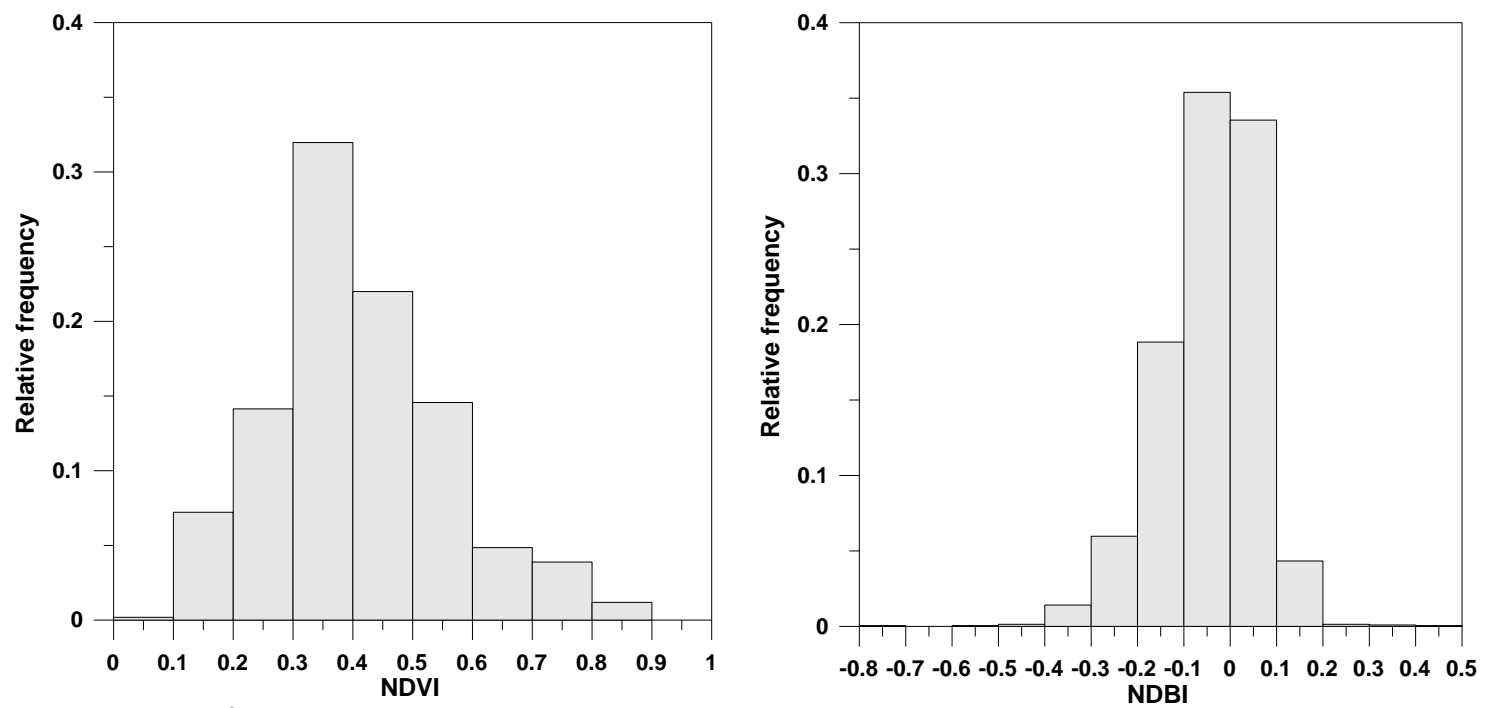

Fuente: Elaboración propia

Citación: SERRA DE LAROCHA, C. et al. Temperatura del aire en la Región Metropolitana de Barcelona a partir de la temperatura en superfície MODIS y de datos topogeográficos. En: Libro de proceedings, CTV 2018. XII Congreso Internacional Ciudad y Territorio Virtual. "Ciudades y Territorios Inteligentes". UNCuyo, Mendoza, 5-7 septiembre 2018. Barcelona: CPSV, 2018, p. 357-368. 
La latitud (lat), longitud (lon) y distancia a la costa o continentalidad (con) se han obtenido a partir de software ArcGIS (Geographic Information Systems, GIS). La altitud (alt), orientación (ori) y pendiente (slp) se han extraido del Modelo Digital del Terreno, MDT, del Institut Cartogràfic i Geológic de Catalunya, ICGC con una resolución de $15 \mathrm{~m}^{2}$

En la Tabla 1 se presenta una comparativa entre los valores medios, mínimos y máximos de toda la base de datos de la RMB con resolución $1 \mathrm{~km}^{2}$ y para el caso de las 48 estaciones seleccionadas. Como puede verse en la tabla las diferencias entre ambas no son muy importantes lo que indica que las estaciones utilizadas para el estudio son una buena representación de todo el territorio de la RMB.

\section{Tabla 1. Comparativa de los valores mínimos, medios y máximos de las variables*}

\begin{tabular}{|c|c|c|c|c|c|c|c|c|c|c|}
\hline Pixels & LSTd & LSTn & $\overline{\text { NDBI }}$ & NDVI & Lat $\left(^{\circ}\right)$ & Lon $\left({ }^{\circ}\right)$ & Con (m) & Alt (m) & Ori $\left(^{\circ}\right)$ & SIp(\%) \\
\hline Media & 23,00 & 11,09 & $-0,09$ & 0,52 & 41,52 & 2,09 & 15565,01 & 294,53 & 165,86 & 23,40 \\
\hline Min & $-13,93$ & $-8,39$ & $-0,57$ & $-0,20$ & 41,19 & 1,47 & 1,92 & 0,00 & 0,00 & 0,00 \\
\hline Max & 48,13 & 30,65 & 1,00 & 0,98 & 41,81 & 2,78 & 41103,86 & 1646,00 & 359,26 & 163,91 \\
\hline Estac. & LSTd & LSTn & NDBI & NDVI & Lat $\left(^{\circ}\right)$ & Lon $\left({ }^{\circ}\right)$ & Con (m) & Alt (m) & Ori $\left(^{\circ}\right)$ & SIp(\%) \\
\hline Media & 23,54 & 11,47 & $-0,04$ & 0,41 & 41,46 & 2,01 & 13313,41 & 279,17 & 148,30 & 10,66 \\
\hline Min & $-3,35$ & $-5,67$ & $-0,75$ & 0,10 & 41,20 & 1,47 & 185,50 & 2,00 & 0,00 & 0,00 \\
\hline Max & 45,31 & 27,19 & 0,44 & 0,85 & 41,77 & 2,76 & 37477,50 & 1668,00 & 360,00 & 75,00 \\
\hline
\end{tabular}

* Para la base de $1 \mathrm{~km} 2$ de resolución y para las estaciones.

Fuente: Elaboración propia

La variable día del calendario, $\mathrm{cd}$, se ha transformado en una nueva variable $\mathrm{cd}^{*}$ para obtener una relación lineal entre la temperatura del aire y el día del año (Janatian et al. 2017). Esta transformación se ha obtenido a partir de la ecuación:

$$
c d^{*}=\cos \frac{2 \pi\left(c d-c d_{\max }\right)}{365}
$$

cdmax es el día del año 2015 con la temperatura diaria media más alta, que en el caso de la RMB estaría en torno al día 200 del calendario o 19 de Julio, después de hacer un ajuste de los valores de todas las estaciones. En la Figura 3 se muestra la dependencia de la temperatura media con el día del calendario, cd, y con la variable transformada $\mathrm{cd}^{*}$.

Figura 3: Dependencia de la temperatura diaria media del 2015 con cd y cd*
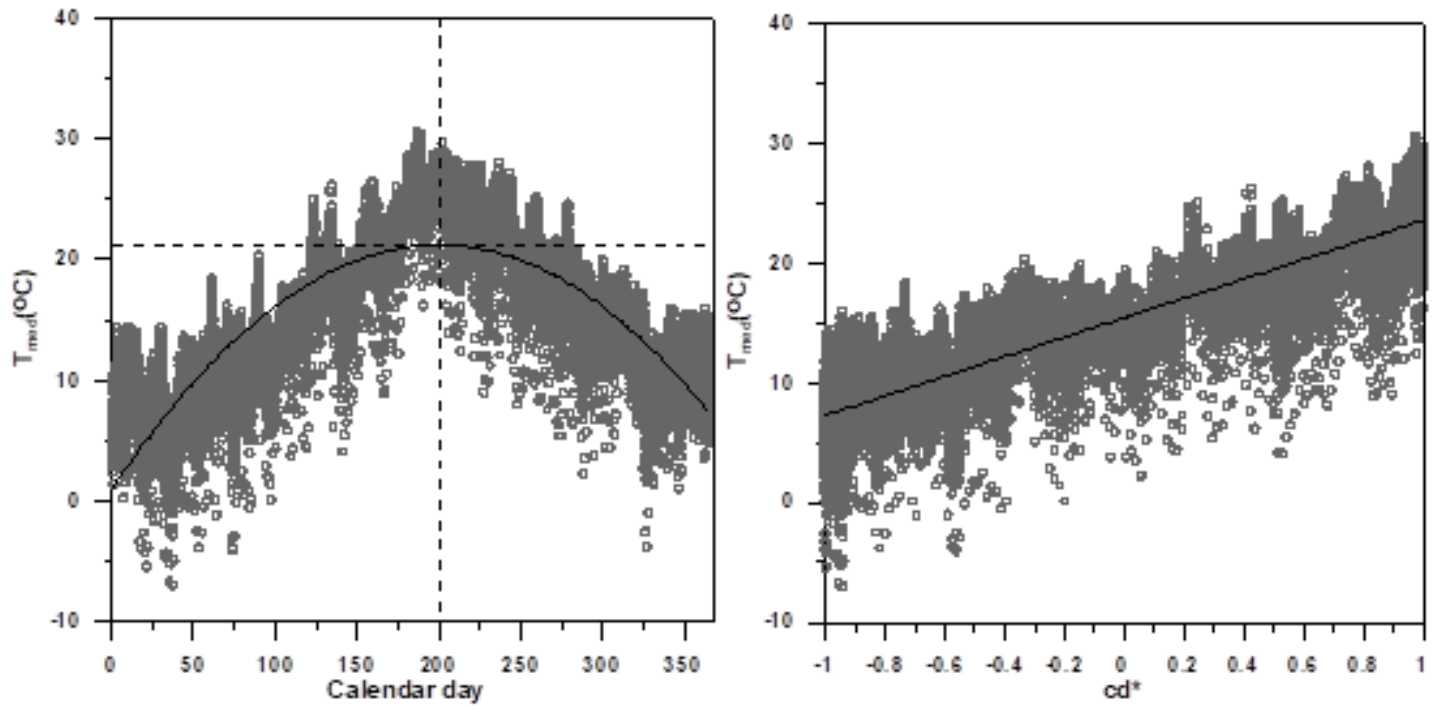

Fuente: Elaboración propia

Citación: SERRA DE LAROCHA, C. et al. Temperatura del aire en la Región Metropolitana de Barcelona a partir de la temperatura en superfície MODIS y de datos topogeográficos. En: Libro de proceedings, CTV 2018. XII Congreso Internacional Ciudad y Territorio Virtual. "Ciudades y Territorios Inteligentes". UNCuyo, Mendoza, 5-7 septiembre 2018. Barcelona: CPSV, 2018, p. 357-368. 
La correlación entre la variable orientación y las temperaturas es muy baja o incluso no significativa. Para mejorar dicha correlación se ha optado por descomponerla en seno y coseno, con lo que han mejorado algo las correlaciones, aunque siguen siendo poco importantes.

\section{Metodología y resultados}

En primer lugar, se calculan las correlaciones de Pearson entre variables con el objetivo de detectar las posibles relaciones entre las temperaturas, mínima, media y máxima del aire con el resto de variables. En la Tabla 2 se muestran dichas correlaciones y, como era de esperar, las más altas se obtienen entre $T_{\min }, T_{\operatorname{med}}, T_{\max }$ y las temperaturas en superficie, $\mathrm{LST}_{d}$ y $\mathrm{LST}_{n}$. También es muy alta la correlación entre las temperaturas del aire con $\mathrm{cd}^{*}$. El resto de correlaciones entre las temperaturas del aire y las variables independientes es inferior a 0.23. También se muestran las correlaciones entre las variables independientes y en algunos casos los valores son medios, como la que existe entre la latitud y la longitud, o entre la altitud y la continentalidad. Las correlaciones no significativas se indican mediante "ns". En segundo lugar, se aplica la regresión múltiple mediante el paquete informático Statistic pachage for Social Sciences, IBM-SPSS. En él se introducen como variables dependientes $T_{\min }$, $T_{\operatorname{med}} \mathrm{O} \mathrm{T}_{\max } \mathrm{y}$ como variables independientes todas las demás, ya que el mismo programa es capaz de eliminar aquellas que no sean significativas, de acuerdo con el valor $P$ y $\alpha=0.05$.

Tabla 2. Correlaciones de Pearson entre las variables

\begin{tabular}{|c|c|c|c|c|c|c|c|c|c|c|c|c|c|c|c|}
\hline & $T_{\max }$ & $\mathbf{T}_{\text {med }}$ & $T_{\min }$ & $\mathbf{L S T}_{d}$ & $\mathbf{L S T}_{\mathrm{n}}$ & IDBI & NDVI & lat & Ion & con & alt & ori $_{\sin }$ & $\operatorname{oi}_{\text {cos }}$ & slp & $\mathbf{d}^{\star}$ \\
\hline $\max$ & 0 & 61 & 1 & ,928 & 3 & 4 & 0 & 27 & 064 & 071 & 229 & 100 & 036 & 112 & 868 \\
\hline$T_{\text {med }}$ & ,961 & 000 & ,968 & ,930 & 967 & 94 & 21 & 39 & ns & 162 & 200 & 042 & 036 & 79 & 897 \\
\hline 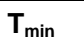 & 881 & ,968 & 0 & 864 & 3 & 3 & 205 & 6 & 070 & 227 & 157 & 018 & 036 & 35 & 874 \\
\hline LST $_{d}$ & ,928 & ,930 & ,864 & 000 & 907 & 247 & 62 & 43 & 43 & 113 & ,204 & 066 & 060 & 5 & 855 \\
\hline $\mathrm{LST}_{\mathrm{n}}$ & ,913 & ,967 & ,953 & ,907 & 0 & 9 & 1 & -,102 & 87 & 203 & 166 & ns & 039 &,- 060 & 302 \\
\hline NDBI & ,214 & 194 & 1 & ,247 & 139 & 000 & 792 &,- 482 & 46 & 066 & -,379 & ,269 & ,257 & 7 & 36 \\
\hline NDVI & 210 & 221 & 205 & 262 & 181 & 92 & 00 & 48 & 255 & 281 & ,524 &,- 300 &,- 291 & 445 & 103 \\
\hline lat & 127 & 139 & 146 & 143 & 102 & 182 & 481 & 1,000 & ,62 & ,502 & ,551 & ,037 &,- 028 & 242, & nc \\
\hline Ion &,- 064 & ns & ,070 &,- 043 & ,087 & 463 & 255 & ,624 & 1,000 &,- 331 & ,034 &,- 110 & ns &,- 011 & $\mathrm{~ns}$ \\
\hline con & 71 & 62 & -,227 &,- 113 & 3 &,- 066 & 281, & 502, &,- 331 & 1,000 & ,599 & ,182 & 037, & ,369 & 110 \\
\hline alt &,- 229 &,- 200 &,- 157 &,- 204 &,- 166 &,- 379 &, 524 & ,551 & ,034 & ,599 & 1,000 &,- 281 &,- 177 & ,435 & ns \\
\hline ori $_{\text {si }}$ & , 100 & ,042 & 018 & ,066 & ns & ,269 &,- 300 &, 03 &,- 110 & , 182 & 81 & 1,000 & ,210 & 7 & ns \\
\hline ori $_{\text {cos }}$ & ,036 & ,036 & ,03 & ,060 & ns & ,257 &,- 291 &,- 028 & ns & ,037 &,- 177 & ,210 & 1,000 & S & רS \\
\hline slp & 112 &,- 079 &,- 035 & 145 & 060 & 357 & ,445 & ,242 &,- 011 & ,36 & ,435 & 87 & רs & 0 & ns \\
\hline $\mathrm{cd}^{\star}$ & 868 & 897 & 874 & 855 & ,902 & 136 & 103 & ns & ns & ns & ns & ns & ns & ns & 1,000 \\
\hline
\end{tabular}

Fuente: Elaboración propia

En la Tabla 3 se muestran los coeficientes beta estandarizados y los no estandarizados de la regresión múltiple. Se destacan en negrita aquellos coeficientes que superan 0.10 . Se comprueba que $\mathrm{LST}_{d}$ interviene en el caso de $\mathrm{T}_{\operatorname{med}} \mathrm{y} \mathrm{T}_{\max }$ pero no en $\mathrm{T}_{\min }$ ya que en éste último caso es no significativa.

Citación: SERRA DE LAROCHA, C. et al. Temperatura del aire en la Región Metropolitana de Barcelona a partir de la temperatura en superfície MODIS y de datos topogeográficos. En: Libro de proceedings, CTV 2018. XII Congreso Internacional Ciudad y Territorio Virtual. "Ciudades y Territorios Inteligentes". UNCuyo, Mendoza, 5-7 septiembre 2018. Barcelona: CPSV, 2018, p. 357-368. 
Tabla 3. Coeficientes beta de la regresión múltiple

\begin{tabular}{|l|r|r|r|r|r|r|}
\cline { 2 - 7 } \multicolumn{1}{c|}{} & \multicolumn{1}{c|}{ Coeficientes estandarizados } & \multicolumn{3}{c|}{ Coeficientes } \\
\cline { 2 - 7 } \multicolumn{1}{c|}{} & $\mathbf{T}_{\min }$ & \multicolumn{1}{c|}{$\mathbf{T}_{\text {med }}$} & \multicolumn{1}{c|}{$\mathbf{T}_{\max }$} & \multicolumn{1}{c|}{$\mathbf{T}_{\min }$} & \multicolumn{1}{c|}{$\mathbf{T}_{\text {med }}$} & \multicolumn{1}{c|}{$\mathbf{T}_{\max }$} \\
\hline Constante & & & & 532.394 & 5.023 & -228.921 \\
\hline LST $_{\mathbf{d}}$ & $\mathrm{ns}$ & $\mathbf{0 . 2 4 5}$ & $\mathbf{0 . 4 1 4}$ & $\mathrm{ns}$ & 0.172 & 0.298 \\
\hline LST $_{\mathbf{n}}$ & $\mathbf{0 . 8 0 1}$ & $\mathbf{0 . 6 6 0}$ & $\mathbf{0 . 4 7 3}$ & 0.786 & 0.660 & 0.484 \\
\hline NDBI & 0.027 & $\mathrm{~ns}$ & $\mathrm{~ns}$ & 1.818 & $\mathrm{~ns}$ & $\mathrm{~ns}$ \\
\hline NDVI & -0.031 & $\mathrm{~ns}$ & 0.057 & -1.481 & $\mathrm{~ns}$ & 2.812 \\
\hline lat & $\mathbf{- 0 . 2 8 6}$ & $\mathrm{ns}$ & $\mathbf{0 . 1 2 3}$ & -13.089 & $\mathrm{~ns}$ & 5.886 \\
\hline lon & $\mathbf{0 . 2 3 4}$ & $\mathrm{ns}$ & $-\mathbf{0 . 1 4 9}$ & 5.015 & $\mathrm{~ns}$ & -3.341 \\
\hline con & 0.096 & $\mathrm{~ns}$ & 0.062 & $5.723 \mathrm{E}-5$ & $\mathrm{~ns}$ & $3.839 \mathrm{E}-5$ \\
\hline alt & 0.083 & -0.043 & $-\mathbf{0 . 1 8 9}$ & 0.002 & -0.001 & -0.004 \\
\hline ori & 0.033 & 0.029 & 0.015 & 0.368 & 0.326 & 0.178 \\
\hline orin & -0.016 & -0.020 & -0.028 & -0.175 & -0.221 & -0.315 \\
\hline sip & 0.047 & 0.022 & -0.014 & 0.023 & 0.011 & -0.007 \\
\hline cd $^{*}$ & $\mathbf{0 . 1 4 4}$ & 0.092 & 0.091 & 1.398 & 0.914 & 0.921 \\
\hline
\end{tabular}

Fuente: Elaboración propia

$\mathrm{LST}_{\mathrm{n}}$ interviene en las tres temperaturas teniendo en $\mathrm{T}_{\min }$ el peso más importante. Después de las temperaturas en superficie, las variables que más contribuyen son la latitud y la longitud para $T_{\min } Y T_{\max }$, ya que para $T_{\operatorname{med}}$ no son significativas. El día del calendario también hay que tenerlo en cuenta sobre todo en el caso de $T_{\min }$ y la altitud en $T_{\max }$. La Figura 4 se representa el diagrama de barras de los coeficientes estandarizados.

Figura 4. Diagrama de barras de los coeficientes estandarizados

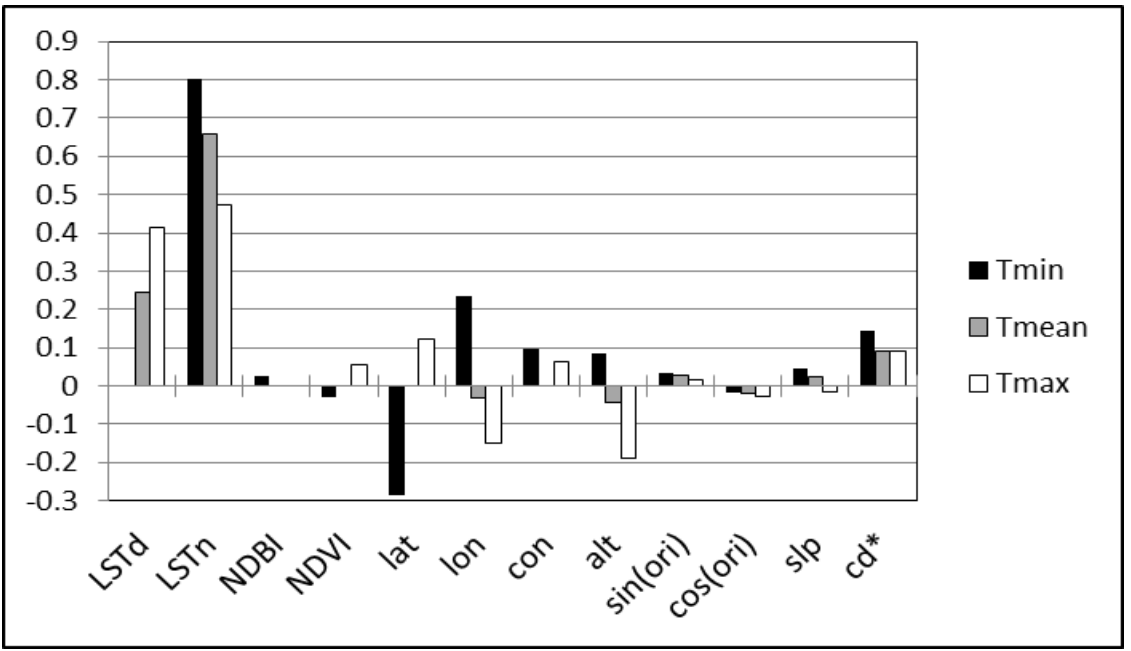

Fuente: Elaboración propia

A partir de los coeficientes obtenidos se han calculado las estimaciones de las temperaturas del aire diarias del año 2015 y se han comparado con los empíricos. Para dicha comparación se ha calculado el error cuadrático medio RMSE y el error absoluto medio MAE:

$$
\text { RMSE }=\left\{N^{-1} \sum_{i=1}^{N}\left(e m p_{i}-e s t_{i}\right)^{2}\right\}^{1 / 2}
$$

Citación: SERRA DE LAROCHA, C. et al. Temperatura del aire en la Región Metropolitana de Barcelona a partir de la temperatura en superfície MODIS y de datos topogeográficos. En: Libro de proceedings, CTV 2018. XII Congreso Internacional Ciudad y Territorio Virtual. "Ciudades y Territorios Inteligentes". UNCuyo, Mendoza, 5-7 septiembre 2018. Barcelona: CPSV, 2018, p. 357-368. 


$$
M A E=\left\{N^{-1} \sum_{i=1}^{N}\left|e m p_{i}-e s t_{i}\right|\right\}
$$

Los valores obtenidos de RMSE son iguales a $1.47,1.95$ y $2.04^{\circ} \mathrm{C}$, y los de MAE de $1.17,1.54$ y $1.62 \stackrel{\circ}{\circ}$.

Se han representado las relaciones entre las temperaturas obtenidas y las observadas $T_{\min }$, $T_{\text {med, }} T_{\max }$ y se acompañan de los coeficientes de regresión al cuadrado, $R^{2}$, para cuantificar la bondad del ajuste (Figura 5). Los valores de $R^{2}$ son de 0.92 para $T_{\min }$ y $T_{\max }$ y de 0.96 para $\mathrm{T}_{\text {med. }}$. En la misma figura también se ha representado el histograma de las diferencias entre los valores estimados y observados en el caso de $T_{\text {med. }}$ En el $51 \%$ de los casos la diferencia es inferior a $1^{\circ} \mathrm{C}$.

Figura 5: Relación entre las temperaturas estimadas y las observadas. Histograma de las diferencias en el caso de $T_{\text {med }}$
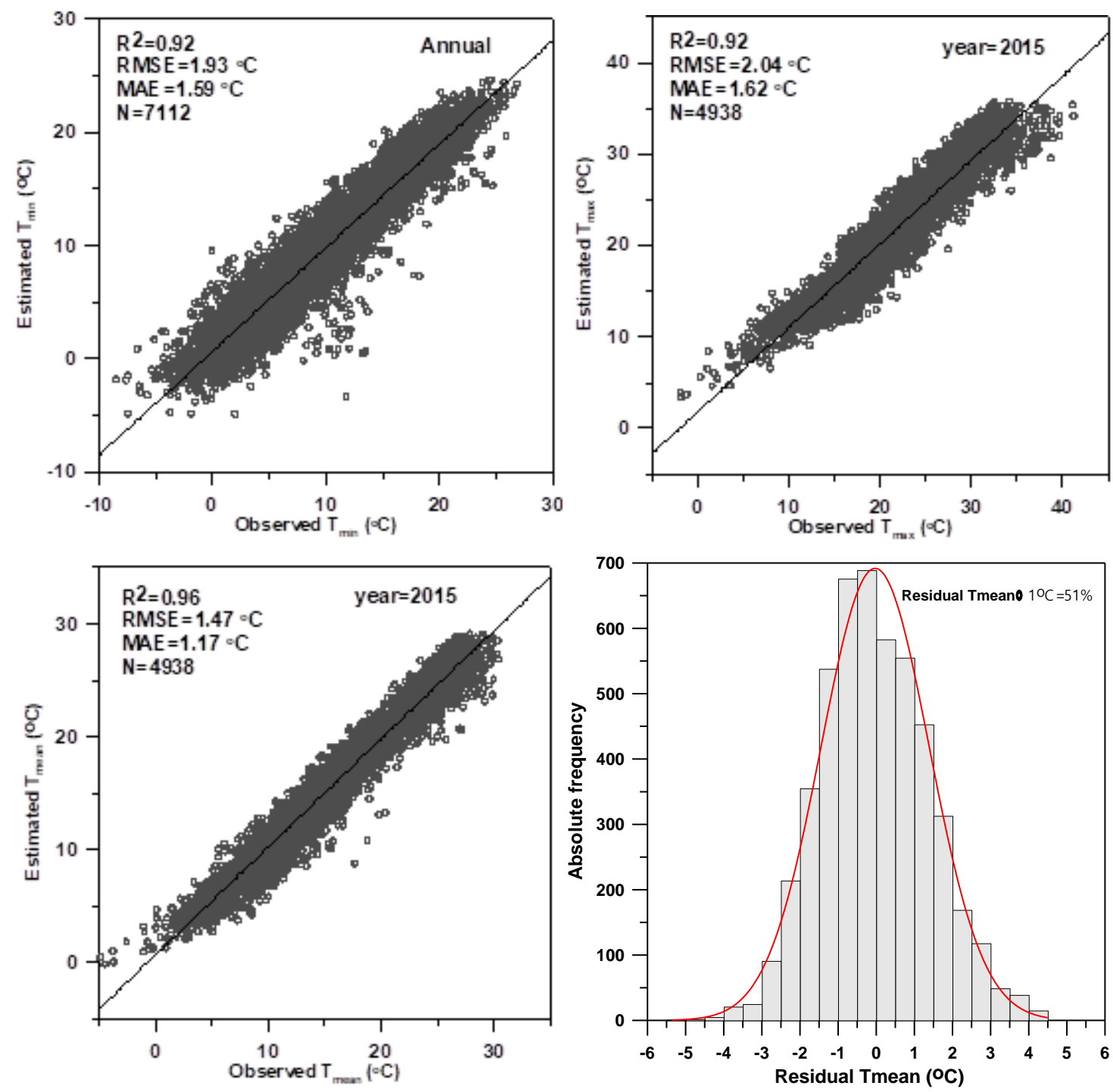

Fuente: Elaboración propia

Citación: SERRA DE LAROCHA, C. et al. Temperatura del aire en la Región Metropolitana de Barcelona a partir de la temperatura en superfície MODIS y de datos topogeográficos. En: Libro de proceedings, CTV 2018. XII Congreso Internacional Ciudad y Territorio Virtual. "Ciudades y Territorios Inteligentes". UNCuyo, Mendoza, 5-7 septiembre 2018. Barcelona: CPSV, 2018, p. 357-368. 
A modo de ejemplo, la Figura 6 muestra los valores de temperatura mínima, media y máxima obtenidos a partir de los coeficientes de la regresión múltiple para el día 29 de noviembre de 2015. Es interesante observar las diferencias de la distribución de temperaturas entre $T_{\min }, T_{\text {med }}$ y $T_{\max }$. En el caso de $T_{\min }$ destacan las temperaturas de la zona costera, sobre todo los máximos observados en la ciudad de Barcelona y en localidades más al sur como Sitges. Queda claro el efecto de isla térmica $(\mathrm{UHI})$ en el caso de $\mathrm{T}_{\min }$, que desaparece en el caso de $\mathrm{T}_{\max }$ donde se observan máximos también en los valles del interior como el del río Llobregat.

Figura 6. Temperaturas del 29/11/2015 calculadas con la regresión múltiple
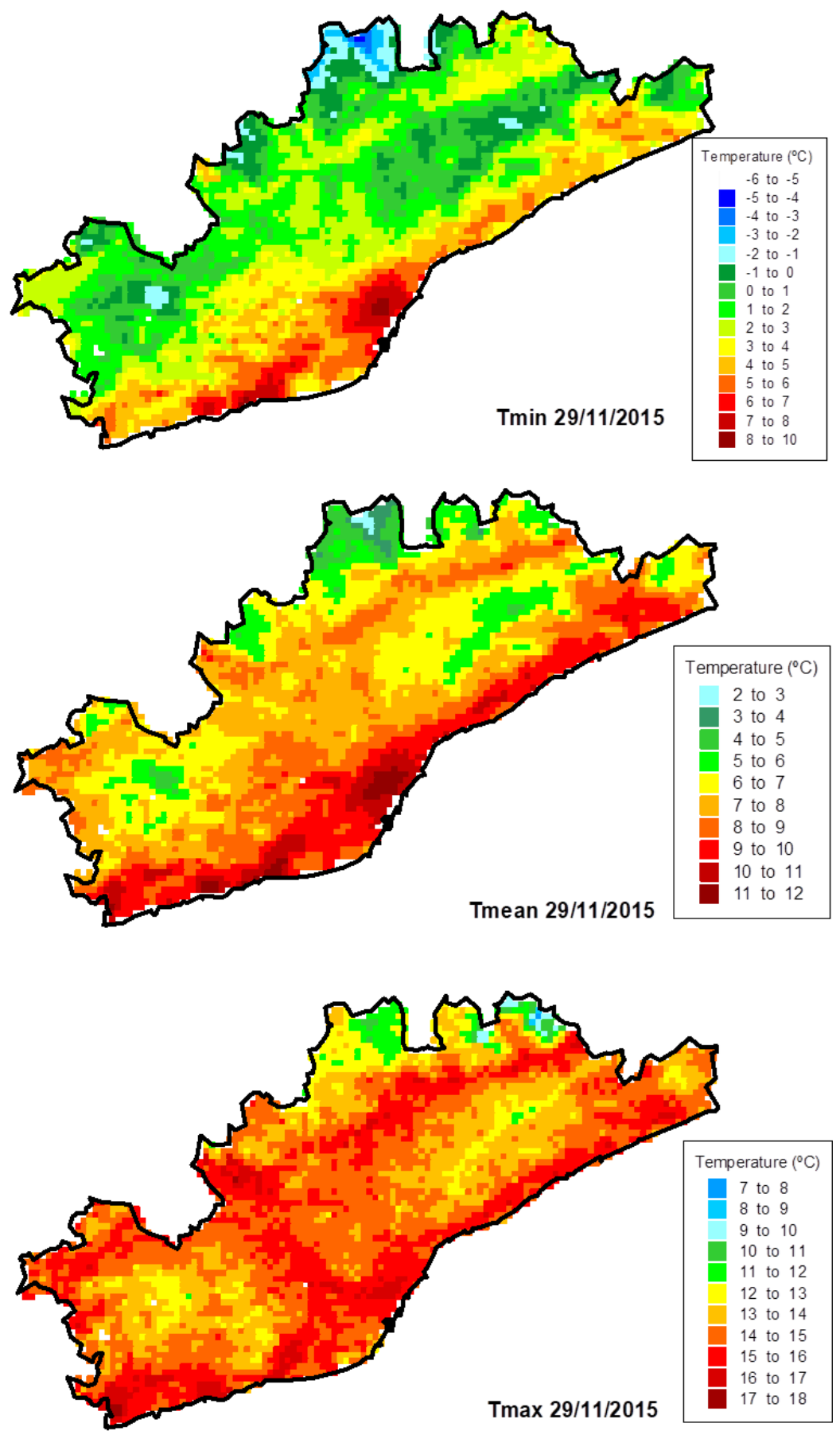

Fuente: Elaboración propia

Citación: SERRA DE LAROCHA, C. et al. Temperatura del aire en la Región Metropolitana de Barcelona a partir de la temperatura en superfície MODIS y de datos topogeográficos. En: Libro de proceedings, CTV 2018. XII Congreso Internacional Ciudad y Territorio Virtual. "Ciudades y Territorios Inteligentes". UNCuyo, Mendoza, 5-7 septiembre 2018. Barcelona: CPSV, 2018, p. 357-368. 


\section{Conclusiones}

Los buenos resultados obtenidos de los coeficientes $R^{2}$ y de RMSE en la regresión múltiple han permitido describir con una resolución de $1 \mathrm{~km}^{2}$ la distribución espacial de temperaturas diarias, mínima, media y máxima, de la RMB. De esta forma, a partir de LST MODIS y de variables topogeogràficas se consigue mejorar la distribución de temperaturas proporcionada por la red termométrica actual. Las variables más relevantes para la estimación de $T_{\min }$ son $L S T_{n}$, latitud, longitud y el día del calendario.

En el caso de $T_{\text {med, }}$ las variables con el coeficiente estandarizado más alto son $L_{S T}$ y $L_{S T}$. En cambio, para $T_{\max }$ hay más variables a tener en cuenta, además de $\mathrm{LST}_{\mathrm{n}}$, $\mathrm{LST}_{\mathrm{d}}$, latitud y longitud también es destacable el coeficiente de la altitud. El resto de variables tienen coeficientes inferiores a 0.10 .

La distribución espacial de la temperatura del aire de forma detallada tiene diversas aplicaciones, como en estudios de cambio climático, agricultura, turismo, seguros, salud y urbanismo, entre otros. En este trabajo, se ha podido detectar la isla de calor urbano (UHI) de la ciudad de Barcelona sobre todo en invierno y en el caso de la temperatura mínima.

\section{Bibliografía}

BENALI, A.; CARVALHO, A. C.; NUNES J. P.; CARVALHAIS, N. y SANTOS, A. Estimating air surface temperature in Portugal using MODIS LST data. En: Remote Sensing of Environment, 124, 108-121. 2012. [En línia] DOI: https://doi.org/10.1016/j.rse.2012.04.024 Disponible en: https://www.sciencedirect.com/science/article/abs/pii/S0034425712002003

BUSTOS, E. y Meza, F. J. A method to estimate maximum and minimum air temperature using MODIS surface temperature and vegetation data: application to the Maipo Basin, Chile. En: Theoretical and Applied Climatology, 120, 211-226. 2015. [En línea] DOI: https://doi.org/10.1007/s00704-014-1167-2

CRISTÓBAL, J.; NINYEROLA, M. y PONS, X. Modeling air temperature through a combination of remote sensing and GIS data. En: Journal of Geophysical Research, 113, D13106, 2008. [En línea] DOI: https://doi.org/10.1029/2007JD009318

FU, G.; SHEN, Z.; ZHANG, X.; SHI, P.; ZHANG, Y. y WU, J. Estimating air temperature of an alpine meadow on the Northern Tibetan Plateau using MODIS land surface temperature. En: Acta Ecologica Sinica, 31 (1): 8-13, 2011. [En línea] Disponible en: https://www.sciencedirect.com/science/article/pii/S1872203210000661 DOI: https://doi.org/10.1016/i.chnaes.2010.11.002

GOOD, E. Daily minimum and maximum surface air temperatures from geostationary satellite data. En: Journal of Geophysical Research Atmosphere, 120 (6): 2306-2324, March 2015. [En línea] DOI: https://doi.org/10.1002/2014JD022438 Disponible en: https://agupubs.onlinelibrary.wiley.com/doi/full/10.1002/2014JD022438

Citación: SERRA DE LAROCHA, C. et al. Temperatura del aire en la Región Metropolitana de Barcelona a partir de la temperatura en superfície MODIS y de datos topogeográficos. En: Libro de proceedings, CTV 2018. XII Congreso Internacional Ciudad y Territorio Virtual. "Ciudades y Territorios Inteligentes". UNCuyo, Mendoza, 5-7 septiembre 2018. Barcelona: CPSV, 2018, p. 357-368. 
JANATIAN, N.; SADEGHI, M.; SANAEINEJAD, S. H.; BAKHSHIAN, E.; FARID, A.; HASHEMINIAA, S. M. y GHAZANFARI, S. A statistical framework for estimating air temperature using MODIS land surface temperature data. En: International Journal of Climatolology, 37, (3): 1181-1194, 2017. [En línea] DOI: https://doi.org/10.1002/joc.4766 Disponible en: https://rmets.onlinelibrary.wiley.com/doi/10.1002/joc.4766

KLOOG, I.; NORDIO F.; LEPEULE, J.; PADOAN, A., LEE, M.; AUFFRAY, A. y SCHWARTZ, J. Modelling spatio-temporally resolved air temperature across the complex geo-climate area of France using satellite-derived land surface temperature data. En: International Journal of Climatology, 37 (1): 296-304, 2017. [En línea] DOI: https://doi.org/10.1002/joc.4705 Disponible en: https://rmets.onlinelibrary.wiley.com/doi/abs/10.1002/joc.4705

NIETO, H.; SANDHOLT, I.; AGUADO, I.; CHUVIECO, E. y STISEN, S. Air temperature estimation with MSG-SEVIRI data: Calibration and validation of the TVX algorithm for the Iberian Peninsula. En: Remote Sensing of Environment, 115 (1): 107-116, enero 2011. [En línea] Disponible en: https://www.sciencedirect.com/science/article/abs/pii/S0034425710002506 DOI: https://doi.org/10.1016/..rse.2010.08.010

PEÓN, J.; RECONDO, C. y CALLEJA, J. F. Improvements in the estimation of daily minimum air temperature in peninsular Spain using MODIS land surface temperature. En: International Journal of Remote Sensing, 35, 5148-5166. Julio 2014. [En línea] Disponible en: https://www.tandfonline.com/doi/abs/10.1080/01431161.2014.935831 DOI: https://doi.org/10.1080/01431161.2014.935831

RECONDO, C.; PEÓN, J. J.; ZAPICO, E. y PENDÁS, E. Empirical models for estimating daily surface water vapour pressure, air temperature, and humidity using MODIS and spatiotemporal variables. Applications to peninsular Spain. En: International Journal of Remote Sensing, 34 (22): 8051- 8080. 2013. [En línea] DOI: https://doi.org/10.1080/01431161.2013.828185 Disponible en: https://www.tandfonline.com/doi/abs/10.1080/01431161.2013.828185

SHEN, S., LEPTOUKH, G. G. Estimation of surface air temperature over central and eastern Eurasia from MODIS land surface temperature. En: Environmental Research Letters, 6, 045206. 2011. [En línea] Disponible en: https://iopscience.iop.org/article/10.1088/17489326/6/4/045206/meta

SOHRABINIA, M.; ZAWAR-REZA, P.; RACK, W. Spatio-temporal analysis of the relationship between LST from MODIS and air temperature in New Zealand. En: Theoretical and Applied Climatology, 119 (3-4): 567-583. 2015. [En línea] DOI: https://doi.org/10.1007/s00704-0141106-2 Disponible en: https://link.springer.com/article/10.1007/s00704-014-1106-2

VANCUTSEM, C.; CECCATO, P.; DINKU, T. y CONNOR, S. J. Evaluation of MODIS land surface temperature data to estimate air temperature in different ecosystems over Africa. En: Remote Sensing of Environment, 114 (2): 449-465. 2010. [En línea] Disponible en: https://www.sciencedirect.com/science/article/abs/pii/S0034425709003113 DOI: https://doi.org/10.1016/..rse.2009.10.002

WENBIN, Z.; AIFENG, L. y SHAOFENG, J. Estimation of daily maximum and minimum air temperature using MODIS land surface temperature products. En: Remote Sensing of Environment, 130, 62-73. 2013. [En línea] DOI: https://doi.org/10.1016/j.rse.2012.10.034 Disponible en: https://www.sciencedirect.com/science/article/abs/pii/S0034425712004221

Citación: SERRA DE LAROCHA, C. et al. Temperatura del aire en la Región Metropolitana de Barcelona a partir de la temperatura en superfície MODIS y de datos topogeográficos. En: Libro de proceedings, CTV 2018. XII Congreso Internacional Ciudad y Territorio Virtual. "Ciudades y Territorios Inteligentes". UNCuyo, Mendoza, 5-7 septiembre 2018. Barcelona: CPSV, 2018, p. 357-368. 
YANG, Y. Z.; CAI, W. H. y YANG, J. Evaluation of MODIS Land Surface Temperature Data to Estimate Near-Surface Air Temperature in Northeast China. En: Remote Sensing, 9 (5): 410, 2017. [En línea] Disponible en: https://www.mdpi.com/2072-4292/9/5/410 DOI: https://doi.org/10.3390/rs9050410

$\mathrm{XU}, \mathrm{Y}$;; KNUDBY, A.; HO, H. C. Estimating daily maximum air temperature from MODIS in British Columbia, Canada. En: International Journal of Remote Sensing, 35, 8108-8121. 2014. [En línea] DOI: https://doi.org/10.1080/01431161.2014.978957 Disponible en: https://www.tandfonline.com/doi/abs/10.1080/01431161.2014.978957?journalCode=tres20

ZAKSEK K. y SCHROEDTER-HOMSCHEIDT, M. Parameterization of air temperature in high temporal and spatial resolution from a combination of the SEVIRI and MODIS instruments. En: ISPRS Journal of Photogrammetry and Remote Sensing, 64 (4): 414-421. 2009. [En línea] Disponible en: https://www.sciencedirect.com/science/article/abs/pii/S0924271609000410 DOI: https://doi.org/10.1016/.isprsiprs.2009.02.006

Citación: SERRA DE LAROCHA, C. et al. Temperatura del aire en la Región Metropolitana de Barcelona a partir de la temperatura en superfície MODIS y de datos topogeográficos. En: Libro de proceedings, CTV 2018. XII Congreso Internacional Ciudad y Territorio Virtual. "Ciudades y Territorios Inteligentes". UNCuyo, Mendoza, 5-7 septiembre 2018. Barcelona: CPSV, 2018, p. 357-368. 